\title{
La Estimulación Supparrenal por la Anoxemia Aguula expresada en la Variación de los Eosinófilos Circulantes
}

\author{
TRABAJO DE LA CATEDRA DE CLINICA MEDICA Y DEL INSTITUTO \\ DE BIOLOGIA ANDINA \\ Director: Prof. Carlos Monge M. \\ Por Jácobo L. Brener Cukier *
}

\section{INTRODUCCION}

Los problemas de la vida en la altitud han suscitado interés y preocupación, e inducido a múltiples estudios a investigadores peruanos y extranjeros. El anhelo de comprensión ha sido tanto del hombre que viviendo allá tiene en equilibrio su fisiología frente al rigor ambiental (18-24), como del que habitando a nivel del mar asciende a las altas montañas, siendo sometido así a una "agresión anóxica", contra la que tiene que luchar (18).

Con el desarrollo de nuevas técnicas ho sido posible medir en el hombre ciertos cambios en la función adrenocortical y que caen aún dentro de límites fisiológicos (6). Esto ha hecho posible el conocimiento de la intervención de esta glándula en las siiuaciones en que el organismo se ve expuesto a agentes que le exigen esfuerzo, (stress) (15).

También la anoxia aguda causada por la ascensión a la altitud es una situación de emergencia y que por lo tanto exige de la glándula suprarrenal una mayor actividad.

Hemos tratado de observar esto último por la variación del número de eosinófilos circulantes, que es una de las mejores pruebas para investigar la función suprarrenal en el hombre.

* Agradezco a los Profesores Honorio Delgado. Alberlo Hurtado, Benjamín Altia. lel y Manuel Bocanegra por sus consejos en la elaboración de esle trabajo y al Dr. Carlos Monge Cassinelli que nos facilitó el Laboratorio do Investigaciones de la Cáledra de Clínica Médica del Profesor C. Monge. 
Para completar el estudio hemos tratado de observar los valores normales de eosinófilos en nuestro medio (a nivel del mar) y su fluctuación en un período de 4 horas, comparándolo con la variación en la altitud.

\section{PRIMERA PARTE}

UBICACION DEL RECUENTO DE L.O EOSINOFILOS ENTRE LAS PRUEBAS DE FUNCION SUPRARRENAL

Desde que se tiene un meior conocimiento de la acción de a!gunas hormonas suprarrenales y de la hormona adrenocórticotropa hipofisaria que estimula su secreción, el estudio de estas glándulas se hace intensivamente.

Debido a las grandes repercusiones que sobre los procesos metabólicos producen las hormonas suprarrenales, los dosajes de diferentes componentes de la sangre y de la orina pueden ser un reflejo relativo de la actividad adrenal. Las diferentes hormonas suprarrenales en líneas generales pueden ser agrupadas desde un punto de vista fisiológico (no estrictamente químico) en tres tipos hormonales de acción diferente (20).

Tipo A.-Regula el metabolismo salino y acuoso, a través de su acción sobre los riñones (mineralocorticoides de Selye) (35).

Tipo B. - Es anabolisador de proteinas, medianamente androgénico y responsable del crecimiento piloso axilar y pubiano en la mujer (hormona "N" de Albright) (1).

Tipo C. Estimula el calcobolismo protéico, causa significativa r.soglucogénesis y está asociado en alguna forma con la resisteicia específica o no escecífica a las enfermedades. Produce linfocitolisis y eosinofilolisis con aumento de las inmunoglubulinas (hormona "S") de Albright (1) y glucocorticoide de Selye (35).

Hasta la actualidad no exisie una prueba enteramente satisfactoria para medir la actividad secretora de la corteza adrenal. Sin émbargo, se han hecho una serie de indices para avaluar esa 
actividad, que siguiendo muy de cerca y casi constantemente a Sayers (33), se los clasifica de la siguiente manera:

1.-Por las alleraciones en la corloza adrenal.

A.--Análomo-macroscópicamente: Hay una buena relación entre el peso cadrenal y la actividad de esta glándula. En ol stress severo, la glándula, además de aumentada de volumen, aparece también de color marrón rojizo, en lugar de amarillo, debido a la pérdida d: los lípidos citop!asmáticos.

B.--Hislológicamenle:

1.-Lípidos (con linción por el Sudán preferentemente, o cor algún otro colorants). En genersl, su baja indica hiper-actividad y la acumulación de lípidos hipoactividad.

2.-Localización de las zonas secretoras:

Glomerular

Fasciculada

Reticular

Las diferenles zonas pueden inostrar una acumulación o baja do lípidos, en grado variable al responder el Stress.

Se observa una respuesta mencr en la glomerular al estimular la glándula con ACTH.

3.--Kelosleroides: Los hisloquinicos aum no están en condi. ciones de identificar o localizar a los esteroides en la corteza adrenal.

C.-Quinicamenle:

1.-Colesterol: Cuando la adrenal está inactiva contiene 5\% de su peso en colesterol; más que cualquier otro tejido. Disminuye cuando aumenta la actividad secrelora cidrenal. En la ausencia de ante-hipófisis, el colesterol adrenal es inerie al stress, y usualmente está en concentración mayor que lo normal. Este fenómeno, 
junto con el hecho de que el colesterol está estrechamente relacio. nado químicamnte a los esteroides corticales, sugieren fuertemente que el colesterol sea un precursor de las hormonas corticales.

2. - Acido ascórbico: En general hav una relación inversa entre la actividad adrenocortical y la concentración del ácido ascórbico adrenal. En ausencia de la antehipólisis el ácido ascórbico normal no se afecla por el stress. Su papel en los procesos matabólicos adrenales es desconocido.

3.-Fósloro: La utlidad del lósloro radioaclivo como elemer. to de valor en la investigación de la actividad adrenal aún no está esclarecido; es un problema actualmente en eshidio.

II.-Por las alleraciones en el organismo.

A. - Lintocitos: La determinación de los linfocitos circulantes es un elemento de valor en la evaluación de la actividad adrenocortical. Dougherty y White con sus estudios sobre la accićn de la corteza adrenal en relación a los linfocitos (7), han hecho po. sible su utilización como elemento de prueba. Se observa una linfocitosis en los animales adrenalectomizados y en pacientes con enfermedad de Addison, lo que probablemente refleja un bajo nivel de las hormonas adrenocorticales. Sin ermbargo, hay que tener en cuenta que pueden producir linfocitopenia los Rayos X, $y$ el Uretano, que tienen una acción linfocitolítica directa. Una descarga esplénica de linfocitos puede oscurecer la linfocitopenia del stress, bajo ciertas condiciones es ésta la probable razón porque la linfocitopenia que se produce después de la inyección de epinefrina es precedida por una linfocitosis (33).

El mecanismo de la linfocitopenia cuando se inyecta hormona adrenotrófica parece consistir en una baja en la liberación de un número adecuado de linfocitos hacia la circulación (7).

B.-Eosinótilos: La observación de que el número de eosinófilos circulantes es regulado por la actividad del sistema adrenocóriticopituitario, ha hecho posible un método muy simple para avaluar la función adrenocortical (33), (39), (32). La prueba 
de los eosinótilos se destaça como el mejor índice de la función suprarrenal (33). Después de la inyección de ACTH, la caída en el número de,eosinófilos, expresada en porcentaje, es aproximáda. mente doble que el de los linfocitos; la respuesta de los eosinófilos es menos variable que la de los linfocitos. Se pueden contar directa y rápidamente sin la necesidad de un recuento diferencial; y con la secreción continuada de esteroides persisten bajos, mientras que los linfocitos después de una caída inicial se elevan pronto a su nivel anterior (32).

Diferentes tipos de stress dan lugar a eosinopenia, enumeraremos algunos: la anoxia inducida (26), (15); la inyección de adrenalina (5); la electroestimulación no convulsiva (38); en la terapia electroconvulsiva (2), (12), (6); en "situaciones de hipoglicemia (27), (3); después de traumas quirúrgicos (32), (11); en hiperglicemias (3); en el infarto de miocardio (8), (11); en la exposición al frío (2l); en los estímulos psicológicos intensos (21).

El test de los eosinófilos, como el de los linfocitos, es de utilidad indudable en el diagnóstico de la insuficiencia adrenocortical total o casi total. El valor de este test en relación al compromiso parcial de la glándula suprarrenal aún no está esclarecido; sin embargo Speirs y Meyer (37), concluyen que la prueba de los eosinófilos es capaz de descubrir aún insuficiencias relativas de las glándulas adrenocorticales en ratones.

Sin embargo los eosinófilos son influenciados por factores diferentes a los de la secreción adrenal. Àsí el número de eosinófilos de los addisonianos se mantiene fijo y no baja con el ACTH o inyectando $0.3 \mathrm{mgr}$. de epinefrina, pero en cambio se produce una eosinopenia cuando estos enfermos hacen una complicación infecciosa. También ocurre una eosinopenia en los addisonianos cuando se les inyecta mayor cantidad de epinefrina; $1.5 \mathrm{mgr}$. (32). En sujetos psiconeuróticos hay quienes afirman que existe diferencia en la respuesta al estimularlos con ACTH (28), (30), pero otros no lo confirman (36).

A la inversa, en procesos alérgicos, cuando la producción excede la posibilidad de destrucción de eosinófilos, el porcentaje de caída en sujetos con glándulas suprarrenales normales es inferior a lo que comunmente se observa (39). Quizá también suceda lo mismo en sujetos parasitados y no como lo preserita Hemández (13). 
El hallazgo de una eosinopenia es de por sí una indicación de la secreción adrenohormonal grandemente aumentada (35). Pura esta regla hay una sola excepción: una muy marcada eosinopenia puede encontrarse en caso de aplasia medular (32).

De todas maneras el les! de los eosinófilos es la medida más: simple y práctica de la aclividad suprarrenal (33).

C.-Melabólicas: La insuficiencia adrenocortical está caracterizada por diversas alteraciones melabólicas que incluyen hipersensibilidad insulínica, poca diuresis después de la administración de agua, deficiente retención de sodio en el ayuno sódico, con aumento del potasio sérico. Pero estas pruebas no sirven para determinar el grado de actividad adrenocortical en un organismo con un sistema pituitario-adrenal intacto.

La deficiente alza de la relación ácido único-creatinina, después de inyectar ACTH indica una insuficiencia adrenocortical.

III.--Esteroides urinarios.

En la orina humana se encuentran sustancias que muestran ciertas propiedades de las hormonas corticales. Se les llama "corticoides", porque son sustancias no claramente identificadas, pero que tienen una o más características de las hormonas corticales. Se les divide en:

A.-Biocorticoides urinarios: Son los que tienen propiedades biológicas similares y que son determinadas por métodos también biológicos. Se les investiga por intermedio de dos propiedades:

1.-La protección al frío "CP".

2.-El depositar glucógeno hepático "LGD".

Los 11 oxisteroides parecen ser los responsables de estas propiedades.

El dosaje de los biocorticoides excretados es relativamente un buen indice de la actividad adrenocortical.

B.-Quimiocorticoides urinarios.-Para su estudio se emplean métodos donde se aprovecha de la solubilidad y de las caracterís- 
ticas químicas de los esteroides corticales. Las siguientes reacciones químicas se aplican "a las diferentes fracciones extraidas de la orina por procedimientos especiales y que contienen los lípidos neutros:

1.-La reducción del ácido fosfomotíbdico "PRL".

2. - La reducción del cobre "CRL".

3.-La generación de formaldehido "FL".

Los quimiocorticoides se hallan en la orina en mayor concentración que los biocorticoides. (Estos determinados por "LGD" y" expresados como equivalentes de la cortisona), debido a que, probablemente, sustancias biológicamente inactivas pueden aparecer en la orina como resultado de la degradación de los córticoesteroi. des activos, en el hígado y en otros tejidos. La desoxicorficoslerona, si está presente en la orina, sería incluida en los quimiocorticoides y no en los biocorticoides.

Hay una buena relación entre los quimiocorticoides urinario: y la evaluación de la actividad adrenocortical.

Hay que recordar, sin embargo, que los esteroides de origen no adrenal contribuyen a la cantidad total de quimiocorticoides en la orina. Los métodos de investigación son relativamenle simple: y convenientes para la determinación rutinaria en el Laboratoric: Clínico.

C.-17-Ketoesteroides: En los sujetos normales, y bajo condiciones ambientales óptimas, la actividad adrenocortical es responsable de los dos tercios de los 17-Ketoesteroides excretados en el hombre y del tolal de los 17-Keloesteroides excretados en la mujer. Los testículos contribuyen con el tercio restante en el hombre.

Los 17-Ketoesteroides urinarios son considerados como un indice de la actividad androgénica de los testículos y de la corteza adrenal y los "corticoides" como un índice de la secreción de las hormonas corticales.

Mientras que Mason y Engstrom (23), dicen que "en el caso individual puede no ser posible descubrir un cambio en la función (córticoadrenal) por medio de una variación en los 17-Ke. toesteroides urinarios", Sayers (33), concluye que "los 17-ketoes- 
teroides urinarios deben ser rechazados como indice de la actividad adrenocorlical, no por lalta de certeza en cuanto a su significación fisiológica, sino por la falk de relación entre los 17-ketoesteroides excrelados y la ciclividad adienocortical, medida por olsos mélodos".

IV.-Corticoides sanguíneos.

A.-Biocorlicoides sanguineos: Canticiades apreciables de hormona cortical se encuentra en la sangre de la vena adrenal. Fn la sangre peritérica, ron los inétodos artuales no es posible des. cubrirlos.

B...-Quimiocorlicoides sanguńneos: Los mélodos empleados en el dosaje de eslos elementos en la orina, no son aplicables aquí, hasta el presente.

Según el anúlisis que acabamos de hacer, después de los corlicoides urinarios son los eosinólilos los que mejor rellejan la aclividad adrenocortical, cuando se trala de investigar sii función on el hombre.

AICWNOS CARACTLRES QUIMICOS DL. LAS IIORMONAS

CORTICOADRENAI.ES

"El lérmino genérico de "Esteroides" lué sugerido por Callow y Younce en 1936, hara el grupo de compuestos biológicamerile imporlantes relacionados a los esteroles vegetales y animales" (23). Los esteroides incluyen a los ácidos biliares, sapogeninas, hormonas sexuales, hormonas adrenocorticales, agluconas ó geninas cardiacas y varios derivados de eslas susiancias.

Todos tiener: en común un núcleo de 4 anillos que consisten de un anillo de ciclopentano unido a un sistema anular fenantrenohidrogenado y llamánidose "ciclopentano-fenantreno". La mayoría de los esteroides tienen grupos metilos los que ordinariamente se señalan por una pequeña raya.

La mayoría de los esteroides aislados de la glándula adrenal pueden ser considerados como derivados del Allopregnane o $\Delta 4$ pregnene. La lórmula estereoquímica y el sistema de numeración es como está en la Fig. $N^{\prime}$ l. 


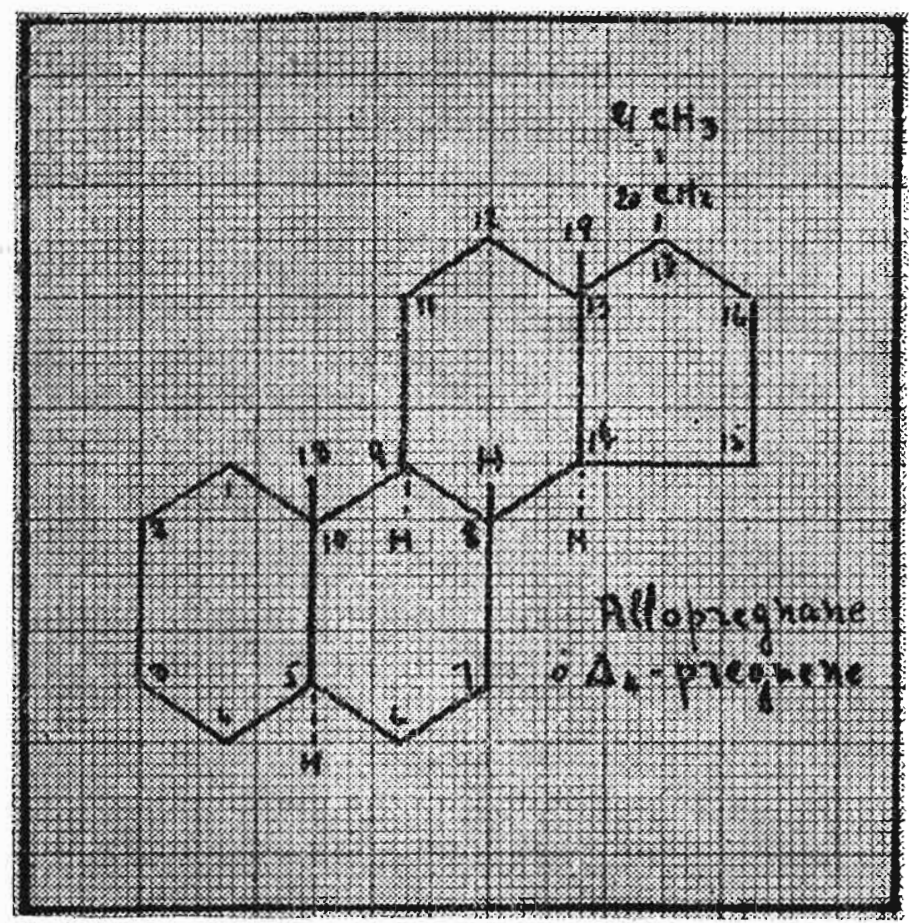

Eig. N? 1

Según ciertas posiciones de los grupos hidróxilo caben dos estéreo-isómeros. Estos se han denominado: aipha y beta; el isómero alpha se traza con un ubicado debajo del plano del anillo considerado. El isómero beta se representa por una línea continua y es considerado como colocado por encima del plano del anillo.

Los esteroides corticales tienen cierlos caracteres que es interesante señalar (34):

1. - Tienen una cadena lateral de 2 átomos de Carbono en C 17.

2.-Un grupo cetónico alpha, beta insaturado es aparentemente ventajoso, pero no indispensable, ya que todos los corticoides activos lo poseen.

3.-Un grupo hidroxílico o acetóxico en $\mathrm{C}_{1 i}$ e es beneficioso, como lo muestra el hecho de la desóxicorticoesterona en relación a la progesterona. 
4.-Un Oxigeno en $C_{11}$ en torma hidraxílica o celónica ha probado ser indispensable para los glucocorlicoides.-Su importan. cia se nota al comparar la curticuenterona con la desoxicorticoesterona, ya que la última difiere de la primera, únicamente en que le falta el oxígeno alcohólico en $\mathrm{C}_{11}$. Esta cérdida conduce a la desaparición de la actividad glixcocorticoide en la desoxicorticoesierona, aunque éste compuesto, es aitamente activo en sus efectos mineralocorticoides.

En la página que sigue figuran algunas hormonas imporlan. les de la corieza adrenal con sus respectivas fórmulas.

De los esteroides corticales, aquellos que poseen un átomo de oxígeno en $\mathrm{C}_{11}$ y $\mathrm{C}_{1}$; ( los llamados $11-17$ oxisieroides) producen vor un mecanismo aún no conocido una caída en la cantidad de us eosinótilos circulanles (32).

MECANISMO DE ACCION DE LA ANOXIA SOBRE IAS GLANDULAS SUPRARRENALES

Para sernos más fácil discutir en alguna forma la acción de la' arıoxia sobre las glándulas suprarrenales, trascribiremos previamente un esquema de Pincus (29), ligeramente modificado. (Ver pág. siguiente).

"Las evidencias indican que el ACT'H es liberado por un neurohumor del hipotálamo. El ACTH es el único agente conocido que acelera la liberación de hormonas adrenocorticales ( $A C H$ ), encima del nivel basal de reposo. Las hormonas corticales ejercen regularmente una acción negativa sobre la pituitaria y quizá también sobre el Sistema Nervioso Central. La ACH liberada es metabolizada en parle a 17-Ketosteroides y corticoides, los que aparecen en la orina. Los órganos finales son: el tejido linfoide y también los múscuios, desde que el Sodio y el Potasio contenido en los músculos $y$ en el cerebro son controlados en parte por el $\mathrm{ACH}$. Los riñones con sus modificaciones en el umbral de excreción para los esteroides, perlenecen lambién al rectángulo rotulado; órgonos finales". 


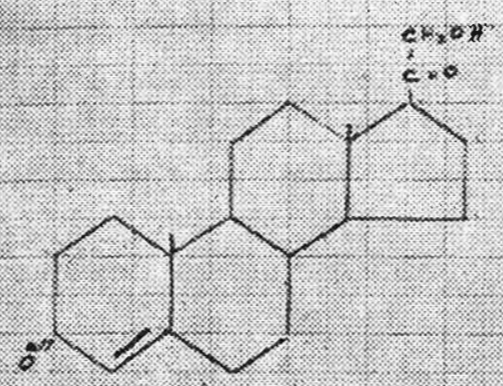

DESOXICOATIKOSTERONR ("DOOC") $Q_{1,}, 4,0,3$.

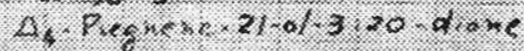

JEHIJROCORTICOSTERONA

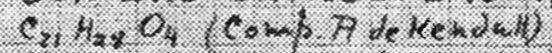

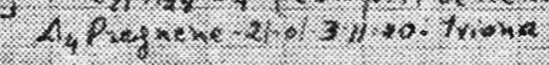

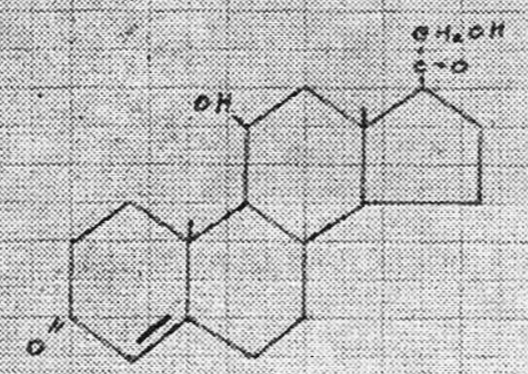

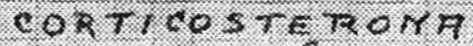

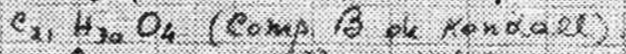

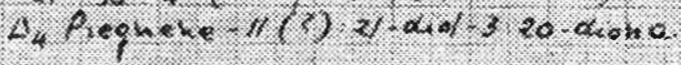

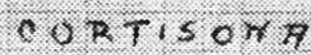

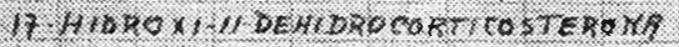

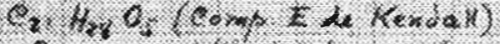

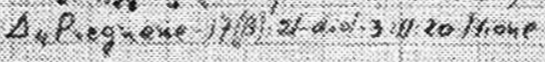

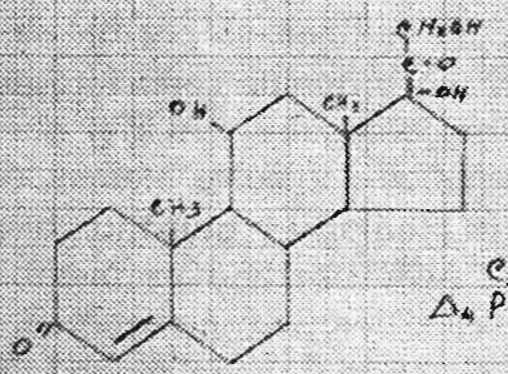

17. HDROXICORTICOSTERONH

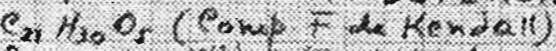

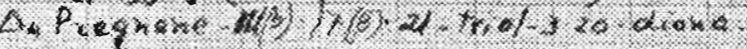




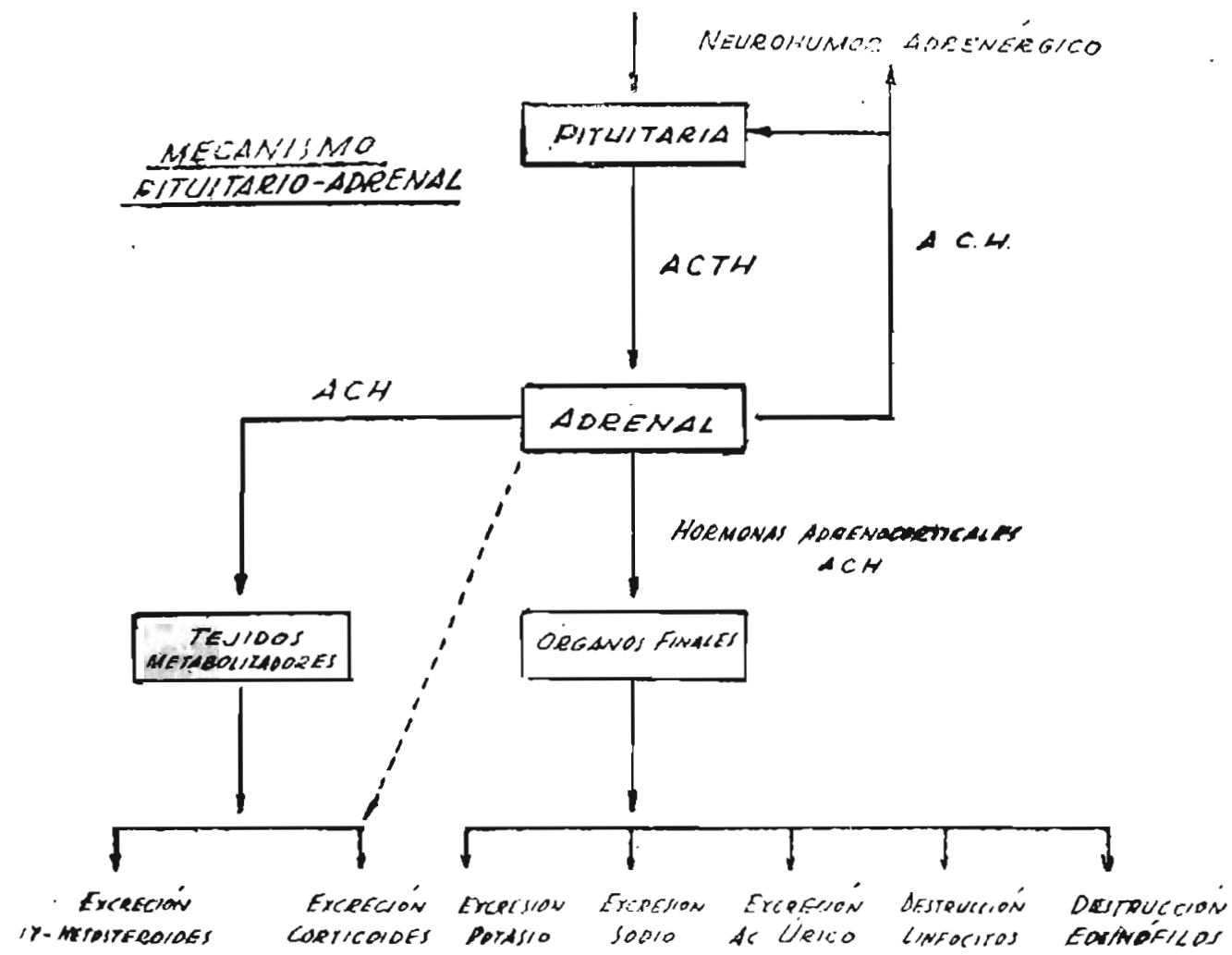

\section{ISQUEMA DE DINCUS (29), LIGERAMENTE MODIFICADO}

La baja tensión de Oxigeno provoca una hipersecreción hormonal de la médula y de la corteza adrenal. La manera como la disminución del Oxígeno cictlia para provocar una hipersecreción de la médula y de la corieza suprarizal cún no está definidamente explicada. Holseay (15), hace lina revisión de las ideas al respecto exislerte: y circe cina la anoxia estimula la secreción de adrenalina por inlermedio aid sistema nervioso central adrenali. no secretor, el que a su vez a través del sistema ortosimpático excita a la médulosuprarenal. Sin embargo, el problema más difícil reside en el mecanismo de secreción de las hormonas córticoadrenales en la anoxia. 
Sabemos que es la córticotrofina hipofisaria la que estimula a la suprarrenal; pero la causa de la descarga de adrenocorticotropina, debida a la anoxia no se conoce bien. "Se ha supuesto que se debe a una acción hipotalámica transmitida por vías nerviosas a la hipófisis, pero ésle no es el único mecanismo posible, pues, diversos agentes provocan la hipersecreción de adrenocorticotropina después de cortar las vías nerviosas hipotálamo-hipofisarias, u obrando sobre la hipófisis injertada. Podría suponerse que la acción hipotalámica se transmita por un agente químico que por los vasos llegue a la hipófisis y la estimule" (15). Otra posibilidad podría ser que la producción de adrenocorticotrofinas se produzca a través del estímulo de la cidrenalina segregada (22), "pero la hipoxia produce descarga de adrenocorticotrofina en ratas $\sin$ médula suprarrenal" (15).

También Long (22), sostiene que las hormonas córticoadrenales podrían disminuir en la sangre al ser consumidas y ésto regularía la secreción de la adrenocorticotrofina. "Por fin podría pensarse que la hipoxia obraría en cierto grado sobre la hipófisis misma y provocar la hipersecreción de adrenocorticotrofina, aunque aún no se ha demostrado este mecanismo" (15). 


\section{SEGUNDA PARTE}

MATERIAL, METODO Y TECNICA

Material de estudio.

Material humano: Los sujetos empleados fueron todos médicos - estudiantes de medicina, cuya edad oscilaba entre los 22 y 32 años. De los 26 sujetos, 17 son mestizos, 8 de raza blanca y 1 amarilla. De todo el grupo sólo 4 fueron del sexo femenino. La gran mayoría eran oriundos de la costa, sólo 2 procedían de Europa (Polonia), pero con una residencia en Lima de más de 15 años; finalmente 3 naturales de la sierra peruana, de altitudes que oscilabom entre 2.000 y 3,500 metros sobre el nivel del mar. Ninguno de los sujetos había ascendido más allá de los mil metros de altitud, desde hacía un año.

Todos eran aparentemente sanos. Entre los antecedentes patológicos se podía anolar, en casi todos, ciertas manifestaciones de alergia, de discreta intensidad, pero que no se hallaban presentes durante los días en que llevamos a cabo el trabajo. También la mayoría presentó parasitosis intestinal en la infancia; en ninguno desde hace 2 años.

Al grupo de personas que fueron llevadas a la altitud les fué practicado además del examen clínico, un estudio radiográfico de pulmones; electrocardiograma y dosaje de hemoglobina, siendo todos los resultados dentro de límites normales. También se hicieron reacciones serológicas (Kahn, Mazzini y Cardiolipina) habiendo resultado positivars en un caso *

Material de laboratorio: Para el recuenio de los ecsinólilos hemos hecho uso de las cámaras especiales de Levy con el rayado de Fuchs-Rosenthal. Sus características son: en primer lugar la profundidad de la cámara que es de $0.2 \mathrm{~mm}$. y con un área rayada de 4 por $4 \mathrm{~mm}$. dando una capacidad total de $3.2 \mathrm{mmc}$. En segundo lugar, el rayado cuyo aspecto en la forma que se ofrece al microscopio es tal como se ve en la Fig. No 2, que corresponde

- Consignamos estos datos, gracias a la gentileza del Dr. Ricardo Cassinelli M. 


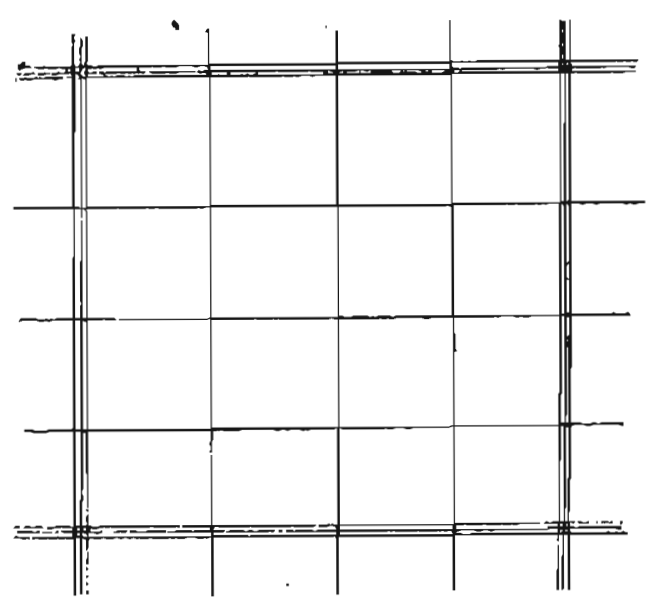

Fig. No 2

a uno de los 16 cuadrados similares que tiene cada cámara. Cada hematímetro posee 2 cámaras. Tanto los hematímetros como las láminas cubreobjetos son certificadas por el "U.S. Bureau of Standards", y que fueron las mismas que se han empleado en todo el trabajo.

Se emplearon 2 pipetas de Thoma (las mismas que se en: plean para la cuenta de leucocitos), también certificadas por el "U.S. Bureau of Standards" y que fueron constantemente las mismas.

El colorante empleado fué la eosina, ideado por Dunger y modificado por Thom (4), en la siguiente dilucićn:

Eosina acuosa al $2 \% \ldots \ldots \ldots \ldots \ldots \ldots$.
Acetona $\ldots \ldots \ldots \ldots \ldots \ldots \ldots \ldots \ldots$
Agua destilad.a c.s.p. $\ldots \ldots \ldots \ldots \ldots \ldots$

Se tilira y debe conzervarise en la retrigeradora, pudiéndose emplear hasta un mes después de preparado.

Lugat de estudio.--Lima, que consideramos a nivel del mar, y Morococha que se halla a 4,540 m. sobre el nivel del mar, con una presión barométrica de $453 \mathrm{~mm}$. Hg. La saturación de oxígeno es de $78.9 \pm 0.68 \%$, a las 2 horas del arribo (16). 
Método.-El grupo de personas llevadas a la altitud, fueron solicitadas a tener un período de sueño de 7 o más horas, en la noche anterior al viaje. Asimismo, abstenerse de ingerir bebidas alcohólicas y a tener situaciones emocionales intensas. Fueron recogidas en automóvil desde su domicilio, entre las 4 y 5 a.m. el día de la subida, no sometiéndoseles a esfuerzos físicos de con. sideración. A esta hora fué extraída la primera muestra para el recuento, que fué en ayunas, permitiéndoseles después tomar el desayuno y alimentos que desearan.

El viaje comenzaba entre los 8 a.m. y 9 a.m. y la llegada a Morococha se efectuaba entre la 1 p.m. y las $2 \mathrm{l} / 2$ p.m. Las muestras de sangre en Morococha fueron extraídas en algunos de los sujetos en la la. hora del arribo, en otros en la $2 a$, en la $3 a$. o en la $4 a$. hora. Se practicó después el examen clínico correspondiente.

A los sujetos empleados para el recuento simple de eosinófilos en Lima, les fué extraída la muestra de sangre entre las 8 y 9 a. m., en ayunas, para la investigación de los valores normales. En algunos, se extrajo también una muestra a las 4 horas, simulando un "Test de Thorn" (39), pero sin inyectar ninguna droga. En este caso, inmedlatamente después de extraída la primera muestra, el sujeto tomaba su desayuno, tal como se aconseja en dicho test.

Se empleó sangre venosa extraída por lo general de una de las venas de la flexura del codo; se tuvo el cuidado de que el brazo no estuviera ligado por más de 2 ' antes de ser punzada la vena. Se usó heparina como anticoagulante, una goła para los 3 a 5 cc. de sangre. Todo el material usado para la extracción y depósito de la muestra estaba estéril y seco. La sangre se empleaba dentro de las 4 horas de la extracción, para el recuento. En esie sentido, no hemos observado mayor variación entre el recuento inmediato y el efectuado 4 horas después, en algunas muestras. Está esto de acuerdo con la uniformidad en el recuento de las muestras dejadas hasta 24 horas a la temperatura ambiente o en refrigeradora. hallado por Weiner y Morkovin (40). Esto les hace confirmar el hecho que el eosinófilo es el elemento más resistente de los granulocitos, pudiendo vivir de 10 a 14 días.

El aspecto de los eosinófilos es de un rojo oscuro contra un fondo rosado; los demás leucocitos no se tiñen. 
Para los cálculos estadísticos y gráficas hemos seguido los "Métodos Estadísticos" del Dr. A. Hurtado (17).

\section{TECNICA}

Hemos hecho el recuento de eosinófilos en cámara, por ser el más exacto, ya que el recuento diferencial, según se refleja er la Hiteratura, deja un gran margen de error (4). (31). El método de coloración empleado ha sido el ideado por Dunger y modiffcado por Thorn (4). Haremos una breve descripción de la forma cómo hemos procedido.

Después de agitar durante tres minutos la muestra de sangre, se hacía la aspiración en una de las pipetas de Thoma hasta la marca 1.0, aspirándose luego el colorante hasta la marca 11; igual cosa en la otra pipeta. En seguida se agitaban suavemente durante 30 segundos y se llenaban con cada pipeta 2 cámaras, dejándolas reposar durante 3 minutos, haciendo luego el recuento que debía terminar antes de los 20 minutos. En caso que durara más, se hacia un nuevo recuento de la misma muestra en un hematímetro (ya que por lo general el primero estaba dentro del tiempo límite). El recuento se hacía en las 4 cámaras y de todos los eosinófilos allí existentes, multiplicando el resultado por la constante 0.781 , lo que arrojaba el número de eosinófilos por milímetro cúbico. Esta constante se obtiene del siguiente raciocinio:

Sabemos que $3.2 \mathrm{mmc}$. es el volumen de cada cámara y como hemos hecho el recuento en 4 cámaras tendremos un volumen total de $12.8 \mathrm{mmc}$. Al haberse hecho la dilución al décimo, entonces es $1.28 \mathrm{mmc}$. de sangre que hemos empleado y para saber cuántos eosinófilos hay en un mmc. debemos dividir la cifra de eosinófilos hallada entre 1.28 o en su defecto multiplicarla por 0.781 que resulta de dividir la unidad entre 1.28.

\section{RESULTADOS OBTENIDOS}

1.-Cifras normales de eosinótilos circulantes.

Se efectuó el recuento de eosinófilos en ayunas, de 8 a 9 a.m., a 25 sujetos aparentemente normales. Enumeramos las cifras en 
el cuadro $N^{Q}$ l. En el cuadro $N^{9} 2$ se hallan los resultados finales del análisis estadístico de dichas cifras. La representación gráfica se puede observar en el histograma $\mathrm{N}^{\circ}$ l.

CUADRO NO 1

Cifras normales

\begin{tabular}{l|c|c|c}
\hline & $\begin{array}{c}\text { Eosinótilos } \\
\text { por mmc. }\end{array}$ & & $\begin{array}{c}\text { Eeosinótilos } \\
\text { por mmc. }\end{array}$ \\
\cline { 2 - 3 } H. P. & 70 & R. J. & 139 \\
M. R. & 75 & E. H. & 141 \\
J. B. & 87 & C. M. & 149 \\
D. S. & 95 & V. H. & 155 \\
M. E. & 98 & M. Ch. & 157 \\
Y. S. & 98 & J. B. & 189 \\
A. S. & 100 & H. C. & 195 \\
J. N. & 102 & N. G. & 284 \\
B. A. & 114 & J. V. & 315 \\
J. V. & 121 & D. P. & 344 \\
R. B. & 122 & S. S. & 366 \\
R. C. & 122 & I. Z. & 372 \\
M. B. & 129 & & \\
\hline
\end{tabular}

CUADRO № 2

\begin{tabular}{l|c|c|c|c|}
\hline & Media \pm E.S. & Dsv. St. \pm E. S. & Ooet. Var. \% & Val. Ext. \\
\hline Eos. x mmc. & $165.56 \pm 18.73$ & $91.63 \pm 13.24$ & 55.34 & $70-372$ \\
\hline
\end{tabular}

2. - Variación de los eosinótilos en 4 horas.

En 16 sujetos gozando de aparente salud, se hizo en ayunas de 8 a 9 a.m. extracción de sangre para un recuento de eosinófilos. Se invitaba entonces al candidato a tomar el desayuno como de costumbre, y a las cuatro horas de la primera extracción se hizo una nueva (antes de haber almorzado) para el segundo re- 
cuento. En esto hemos tratado de imitar las condiciones en las que se practica el Test de Thorn *.

En los cuadros Nos. 3 y 4 , se demuestran los resultados oblenidos, y en el histograma No 2, está la representación gráfica:

\begin{tabular}{c|c|c|c|c}
\hline & \multicolumn{5}{c}{ CUADRO No 3 } \\
\hline & $\begin{array}{c}\text { Cifra } \\
\text { basal } \\
\text { Eos. } x \text { mmc. }\end{array}$ & $\begin{array}{c}\text { Después } \\
\text { de cuatro hs. } \\
\text { Eos. x mmc. }\end{array}$ & $\begin{array}{c}\text { Variación } \\
\text { absoluta }\end{array}$ & $\begin{array}{c}\text { Variación } \\
\text { porcentual }\end{array}$ \\
\hline 1 & 189 & 196 & +7 & +3.70 \\
2 & 195 & 201 & +6 & +3.07 \\
3 & 141 & 138 & -3 & -1.64 \\
4 & 122 & 118 & -4 & -3.28 \\
5 & 102 & 93 & -9 & -8.83 \\
6 & 87 & 77 & -10 & -11.50 \\
7 & 149 & 130 & -19 & -12.76 \\
8 & 70 & 60 & -10 & -14.29 \\
9 & 100 & 85 & -15 & -15.00 \\
10 & 961 & 715 & -246 & -25.60 \\
11 & 139 & 102 & -37 & -26.41 \\
12 & 75 & 54 & -21 & -28.00 \\
13 & 157 & 100 & -57 & -36.31 \\
14 & 366 & 226 & -140 & -38.26 \\
15 & 122 & 75 & -47 & -38.53 \\
16 & 114 & 64 & -50 & -43.86 \\
\hline
\end{tabular}

CUADRO No 4

\begin{tabular}{l|c|c|c|c}
\hline Media \pm E. S. & Dsv. St. \pm E. S. & Coef. Var. \% & Val. Ext. \\
\hline Var. Porc. & $-18.59 \pm 3.89$ & $15.07 \pm 2.75$ & .81 .06 & $+1-3.70 a-43.86$ \\
\hline
\end{tabular}

- El Test de Thorn mide "la reserva funcional de las glándulas córticoadrenales". Se practica en la forma que hicimos esta investigación, pero que inmediatamente de extraída la primera muestra se hace una inyección de 25 mgr. de ACTH (Hormona adrenocórlicotropa). Normalmente se produce a las 4 horas una caída de los eosinófilos a más del $50 \%$. 
3. - Variación de los eosinótilos en la anoxemia aguda.

Constituye esto la parte fundamental del presente trabajo.

En la maryoría de los 21 sujetos llevados a la altitud, hemos hallado un notable descenso en el número de los eosinófilos circu. lantes. Las cifras individuales obtenidas, están señaladas en el cuadro No 5 . En el cuadro No 6 , hemos apuntado los resultados del análisis estadístico, y la distribución de los datos están en el histogroma No 3.

Excepto uno, todos los demás sujetos mostraron una evidente caida en sus eosinófilos circulantes, on la altitud. Pero a pesar de las instrucciones dadas, dos sujetos no las siguieron muy bien, habiendo ingerido bebidas alcohólicas en la noche anterior a la ida a Morococha. Y lué precisamente uno de ellos el que más bien tuvo un aumento en el número de sus eosinófilos $(+12 \%)$.

Otro sujeto que sólo mostró un descenso de $8 \%$, presenta una eosinofilia marcada que parece deberse a un proceso alérgico (urticarias muy frecuentes). A pesar que tuvo un descenso de 154 eleméntos, sin embargo porcentualmente es poco marcado.

Entre los 21 sujetos que intervinieron en la investigación, cuatro presentaron un cuadro de soroche agudo. Anotamos a continuación la sintomatología que presentaron, indicando entre paréntesis el número de asorochados que sufrieron determinado síntoma:

Trastomo vasomotores: (Palidez (4)

(Moderada cianosis (3)

Trastornos de orden nervioso: (Cefalea (4)

(Náuseas y vómitos (3)

(Mareos (3)

(Somnolencia (3)

("Consancio mental" (3)

Trastomos circulatorios: (Frialdad en las extremidades (4) (Taquicardia (3)

(Arritmia extrasistólica (1)

(Palpitaciones, dolor precordial (2) 


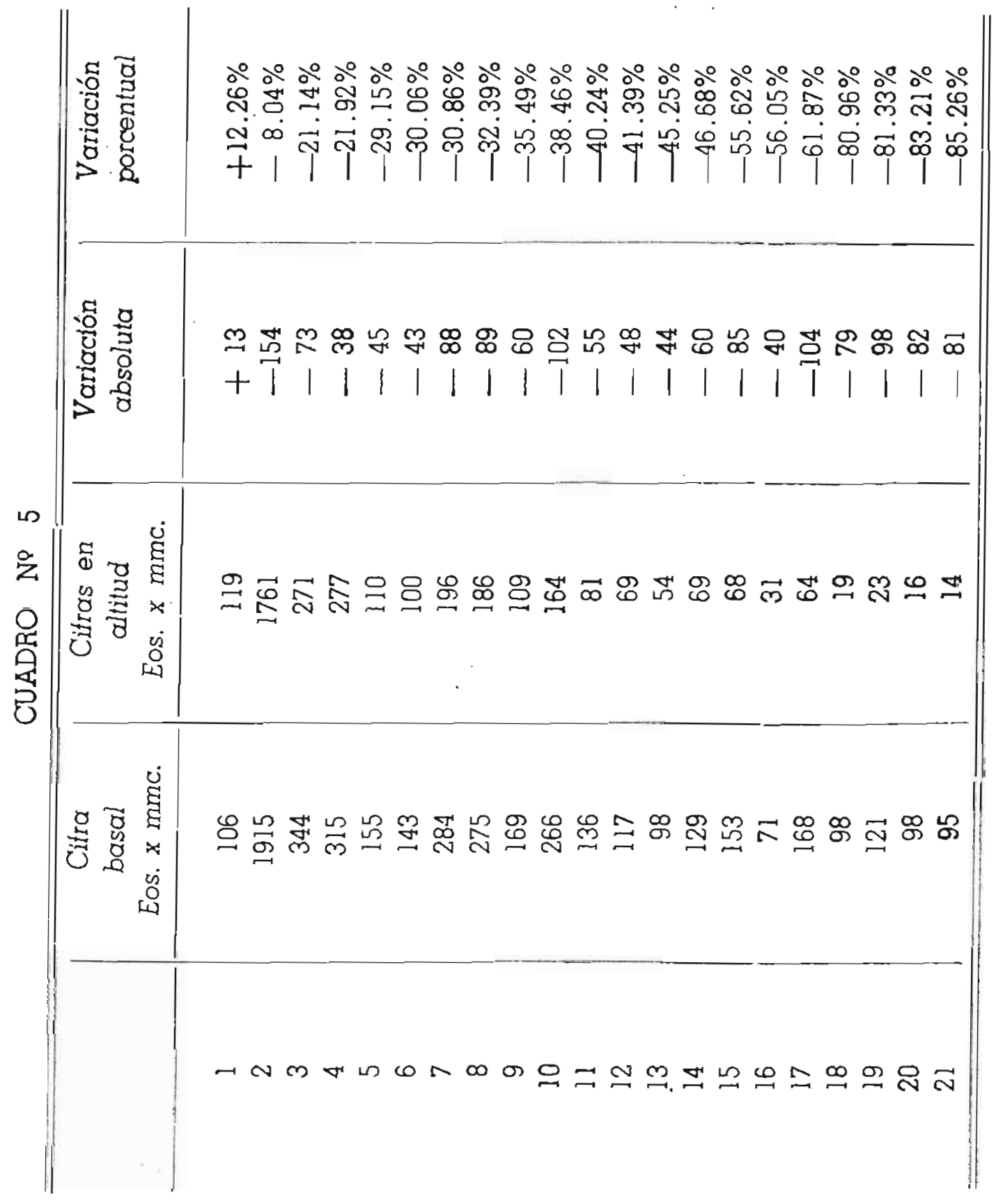


Trastorn. en la func, respir. (Disnea (1)

Trast. en org. sentidos: (Visión de escotomas (2)

Trast. gastrointestinales: (Abalonamiento del vientre (1)

La mayoría de los 17 sujetos restantes presentaron discreta sintomatología que más o menos toleró bien, permitiéndoles ayudar en el trabajo.

Tres de los cuatro sujetos asorochados mostraron un descenso del $80 \%$ de eosinófilos y uno de ellos $30 \%$. Vale decir que el $75 \%$ de los asorochados tuvieron una eosinopenia muy marcada. Entre los 17 sujetos restantes, que no habían hecho un cuadro de soroche agudo, sólo uno mostró descenso tan alto: $81 \%$.

Tratando de observar si hay una diferencia en el descenso de eosinófilos, según el número de horas de estadía en Morococha, hicimos el recuento en un grupo a la hora de llegada, en otro a las 2 horas, a las tres horas y a las cuatro horas. Señalamos los resultados en los cuadros Nos 7 y 8 , y en el histograma No. 4, en el cual las columnas en negro muestran el porcentaje de descenso y las columnas blancas en el centro de aquellas, señalan el número de sujetos empleados en cada grupo. Aparentemente se observa que el mayor descenso ocurrió en el grupo que tuvo una estadía de cuatro horas en Miorococha, pero que puede deberse a que coincldentemente 3 de los cuatro sujetos asorochados están en este grupo, y justamente los tres que presentan el mayor descenso. Si tomáramos sólo en consideración los que no tuvieron el cuadro de soroche agudo, los cuatro grupos presentarian valores promedios de eosinopenias sensiblemente similares: $--36 \% ;-31 \% ;-39 \%$ y $41 \%$, respectivamente.

CUADRO NQ 6

\begin{tabular}{|c|c|c|c|c|}
\hline & Media $\pm E . S$ & Dsv. St. \pm E. S. & Coet. Var. & Val. Ext. \\
\hline Var. Porc. & $-43.48 \pm 55.54$ & $24.80 \pm 3.92$ & $\begin{array}{c}\% \\
57.03\end{array}$ & $+12.26 a-85.26$ \\
\hline
\end{tabular}


CUADRO NQ 7

$\begin{array}{cccc}\text { Cifra } & \text { Cifra en } & \text { Variación } & \text { Variación } \\ \text { basal } & \text { la altitud } & \text { absoluta } & \text { porcentual }\end{array}$

Eos. $x$ mmc. Ébs. $x$ mmc. Eos. $x$ mmc.

GRUPO I De 0 a l hora de estadía en la altitud:

\begin{tabular}{rrrrr}
8 & 275 & 186 & -89 & $-32.39 \%$ \\
11 & 136 & 81 & -55 & $-40.24 \%$ \\
\hline
\end{tabular}

GRUPO Il $D_{\theta}$ l a 2 horas de estadía en la aititud:

\begin{tabular}{rrrrr}
12 & 117 & 69 & -48 & $-41.39 \%$ \\
15 & 153 & 68 & -85 & $-55.62 \%$ \\
5 & 155 & 110 & -45 & $-29.15 \%$ \\
7 & 284 & 196 & -88 & $-30.86 \%$ \\
3 & 344 & 271 & -73 & $-21.14 \%$ \\
2 & 1915 & 1761 & -154 & $-8.04 \%$ \\
\hline
\end{tabular}

GRUPO III De 2 a 3 horas de estadía en la altitud:

\begin{tabular}{rrrrr}
10 & 266 & 164 & -102 & $-38.46 \%$ \\
6 & 143 & 100 & -43 & $-30.06 \%$ \\
17 & 168 & 64 & -104 & $-61.87 \%$ \\
19 & 121 & 23 & -98 & $-81.33 \%$ \\
1 & 106 & 119 & +13 & $+12.26 \%$ \\
\hline
\end{tabular}

GRUPO IV de 3 a 4 horas de estadía en la altitud:

\begin{tabular}{rrrrr}
4 & 315 & 277 & -38 & $-21.92 \%$ \\
16 & 71 & 31 & -40 & $-56.05 \%$ \\
14 & 129 & 69 & -60 & $-46.68 \%$ \\
13 & 98 & 54 & -44 & $-45.25 \%$ \\
20 & 98 & 16 & -82 & $-83.21 \%$ \\
9 & 169 & 109 & -60 & $-35.49 \%$ \\
21 & 95 & 14 & -81 & $-85.26 \%$ \\
18 & 98 & 19 & -79 & $-80.96 \%$ \\
\hline
\end{tabular}

CUADRO NQ 8

Cifra media del descenso de los eosinófilos en la altitud, según las horas de estadía

\begin{tabular}{clc}
\hline Grupo & & $\%$ \\
\hline I & De 0 a l hora de estadía en la altitud & -36.31 \\
II & De 1 a 2 horas de estadía en la altitud & -31.03 \\
III & De 2 a 3 horas de estadía en la altitud & -39.88 \\
IV & De 3 a 4 horas de estadía en la altitud & -56.85 \\
\hline
\end{tabular}




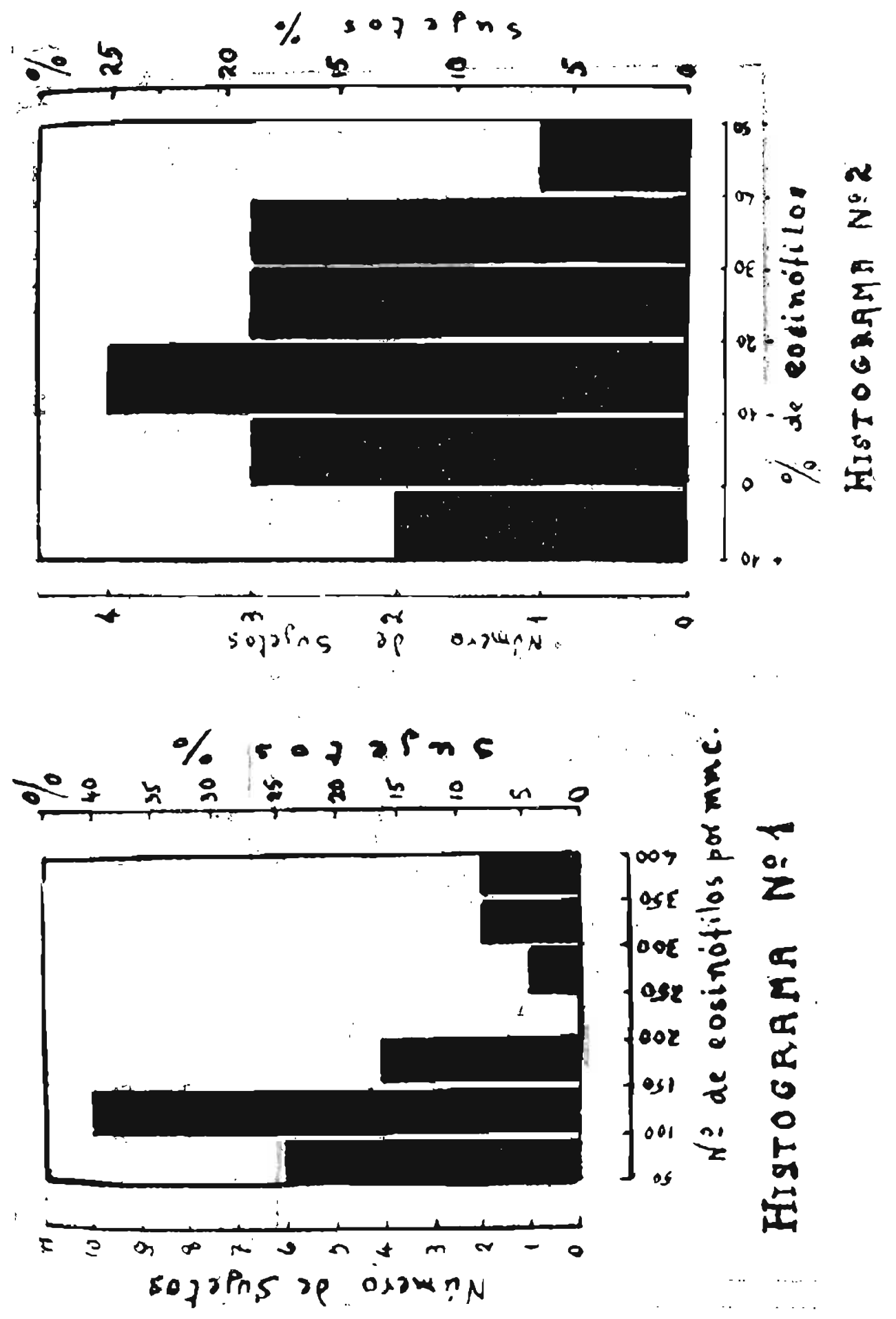



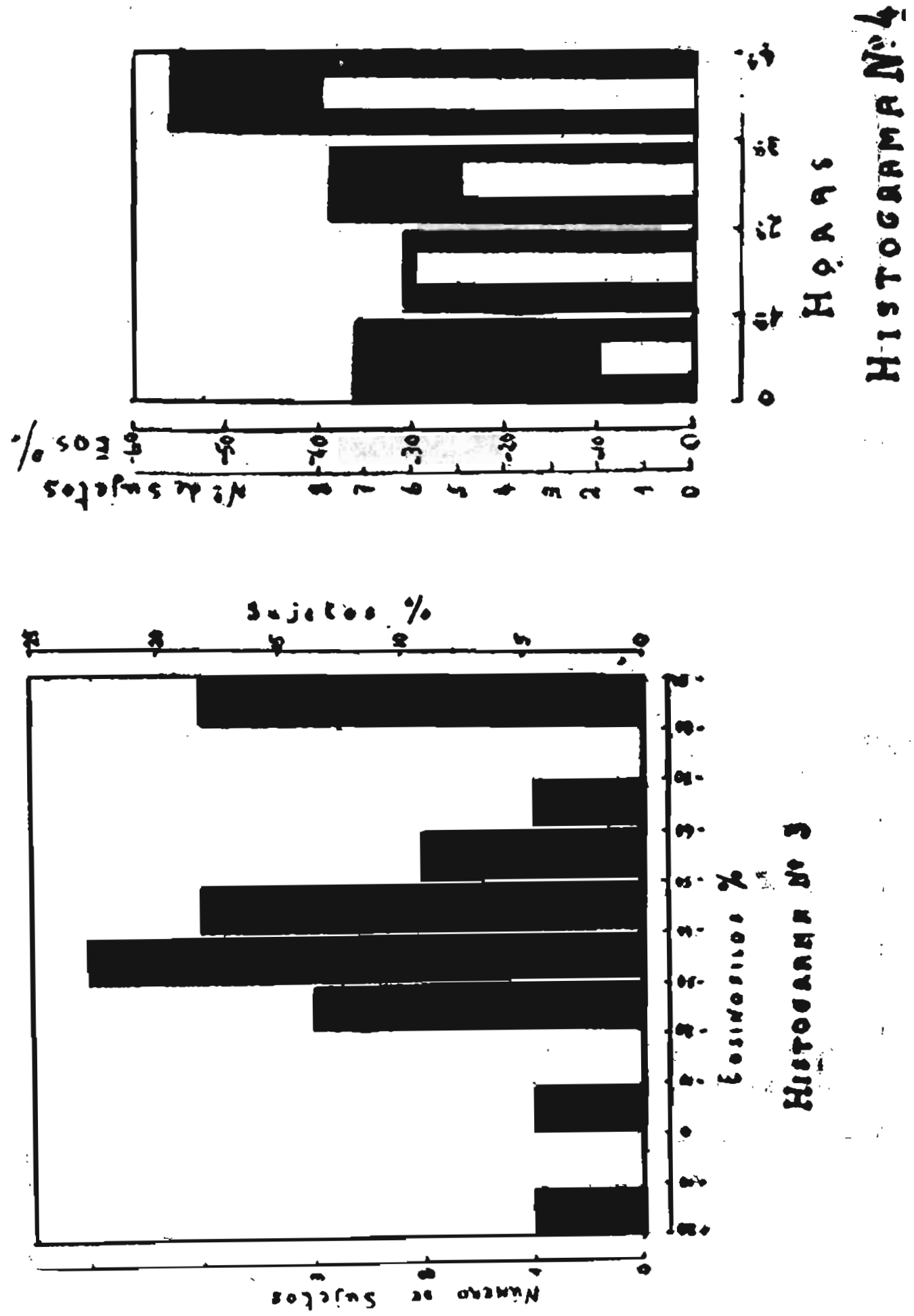
DISCUSION

1. Del Material, Método y Técnica.

Como ya dijimos, hemos empleado el recuento directo de eosinótllos, por ser el más exacto. Puesto que el método del recuento diferencial requiere también un recuento de leucocitos, lo que significa participar de factores de error duplicados, por cada uno de los recuentos. "Los errores de mayor magnitud se hallan en la ivicnica del recuento diferencial. Pueden ser clasificados de acuerdo con Bamelt, como los errores mecánicos que incluyen todas las variaciones en la toma de la muestra de sangre, irregularidades en la distribución de las células y de las áreas sobre las que se hace el recuento; y secundariamente errores accidentales". (31).

En cuanto al método de coloración: se emplean en la actualidad dos métodos: el que nosotros hemos usado, que es el de Dunger modificado por Thorn (4), (39), y el ideado por Randolph (31). Este último sustituye la mezcla eosina-acetona de Dunger por phloxineazul de metileno en glicol propileno. Por este método se puede hacer, simultáneamente también el recuento de leucocitos totales, lo que recomienda su ciutor. Hay quienes opinan que es el método de elección; no lo hernos ensayado por carecer de is droga.

2.-De los Resullados oblenidos:

A. - Cifras normales de eosinófilos.

Las cifras que hemos oblenido, lo fueron en un pequeño grupo humano, por lo que de ninguna manera pretendemos hacer de nuestros resultados un reflejo absoluto de la realidad. Pero sí podemos tener una orientación, ya que las cifras nuestras no se alejan mucho de las que se han obtenido en otras poblaciones humanas. Thorn y asociados (39), dan como cifras normales de eosinótilos de 100 a 250 por mmc. Fisher y Fisher (9), hacen un estudio en 170 individuos, encontrando que sus resultandos están de acuerdo con los hallados por Osgood, Todd y Kracke, siendo sus normales de 10 a 400 eosinófilos por milímetro cúbico. Sola- 
mente alrededor del cincuenta por ciento de sus cifras están dentro de las cifras dadas por Thom. Como se ve en los cuadros Nos. 1 y 2 y en el histograma No l, nuestrac cifras variom entre 70 y 372 eosinófilos por mmc. eslando esto más de acuerdo con las cifras dados por Fisher. El 50 \% de nuestros valores, estarían dentro de las cifras normales dadas por Thorn.

Existe cdemás el problema de las fluctuaciones diarias que deberíase tomar en cuenta al hablar de cilras normales.

B. -Variación de los eosinólilos en cuatro horas:

El hecho de que, en la mayoría de los sujetos se halla encontrado un descenso en el número de los eosinófilos sin el suministro de droga alguna, y tratando de que el sujeto siga dentro do su actividad normal, cotidiana, es muy significativo. En primer lugar, nos habla de la fluctuación del número de eosinófilos, hecho ya conocido (9), (10), y que debe ser tomado en cuenta para quien se ocupa de establecer las cifras normales absolutas. En segundo lugar, nos ínvita a considerar el valor del Test de Thorn. En éste se requiere de la. inyección de hormona adrenocórtico-tropa (ó adrenalina) para estimular por vía exógena là secreción de esteroides córtico-adrenales e investigar el funcionamiento del sistema hipófisis-suprarrenal.

Si los eosinófilos, en estas condiciones, bajan a las cuatro horas más allá del cincuenta por ciento de su cifra basal hablamos de funcionamiento normal (39). Pero nosotros hemos encontrado que sin droga alguna, e imitando las condiciones de Thorn obtenemos un descenso cuya media es de $18.59 \%$ y con valores extremos de $+3.70 \%$ y $-43.86 \%$. Hay quien encuentra variaciones aún mayores y observa que la inyección de extractos córticoadrenales producen cambios en nada diferentes a las variaciones o fluctuaciones diarias normales (9). Sin embargo, nos parece que este hallazgo no altera el valor del Test de Thorn, puesto que esta fluctuación diaria acentuada sólo se observa en sujetos normales, como veremos más abajo.

Flink y Halberg (10), hacen un estudio en 200 sujetos normales, investigando la variación en los eosinófilos que ocurre entre las 6.30 a.m. y 9.30 a.m., observando un descenso de más del $20 \%$ an al $\mathrm{fR \%}$ de las suiatos. A esto llaman "la prueba de eosinopenta 
endógena" y al descenso de más de $20 \%$ lo califican de resultado negativo. Al hacer 41 investigaciones en 20 pacientes que sufren de insuficiencia pituitaria y/ó adrenocortical, observan que 40 pruebas son positivas, es decir, que no alcanzan a un descenso de $20 \%$ o más. Concluyen que resultados negativos con "la prueba dé la eosinopenia endógena" son una evidencia tuerte contra la insuHiclencla pituitaria y/ó adrenocortical.

Nosotros hemos obtenido tests de eosinopenia endógena negativos en solo $43.75 \%$ de nuestros 16 sujetos normales. Pero lo hemos hecho a horas diferentes de los de autores que comentamos. Además, en un cáso (el No 6 del cuadro No 3), lo hemos repetido en otra oportunidad y el resultado fué un descenso de $-56.37 \%$ y no de $-11.50 \%$, como está consignádo. Pero debemos advertir que este sujeto el día que presentó el mayor descenso estaba sometido a intensa actividad mental (se trata del autor del cresente trabajo). No conocemos bien las condiciones en que Flink (10), hace su recuento, pero nos parece importante que las condiciones, tanto de los sujetos presuntos normales, como de los enfermos que se investigan deben ser similares.

C.-Variación de los eosinótilos en la anoxemia aguda:

La anoxia (o como algunos lo llaman la hipoxia), así como otros muchos agentes, constituye un "estímulo de alarma" y de acuerdo a lo que sucede en éstos hay un aumento en la secreción de las hormonas de la corteza adrenal, con los consiguientes cambios metabólicos, citológicos y hormonales que engendran. Hemos tomado uno de los índices más constantes para observar esta actividad suprarrenal y es la variación en los eosinófilos circulantes (35).

Nuestros resultados no coinciden con los obtenidos por Hurtado, Merino y Delgado Febres, que exponen en el magnífico estudio: "La influencia de la anoxemia sobre la actividad hematopoyética". Si bien sus determinaciones no son hechas en Morococha que se halla a 4.540 metros sobre el nivel del mar, sino unas fueron realizadas en Casapalca (4.165 m.), obteniendo una varia. ción de 4.2 eosinófilos por ciento en Lima, a $4.6 \%$ en aquella población. Otras determinaciones fueron hechas en la Cima (4.835 m.), donde encuentran $3.3 \%$ de eosinófilos y en el mismo grupo 
habían encontrado en Lima sólo 3.6\%, notando, pues, variaciones muy pequeñas.

Debemos anotar que el viaje en este último caso, duró para ellos tres y media horas; nuestro viaje tuvo una duración de cuatro a seis horas.

Atribuímos la diferencia, en cuanto a la variación de los eosinófilos, a los distintos métodos empleados y quizá también a que nosotros hicimos el recuento cuando el sujeto estaba sometido a la anoxia durante más tiempo. Ya que las cilras más bajas en el recuento de eosinófilos, después de someter al sujeto a cualquier stress, son de pasadas tres a cuatro horas, aunque desde la primera hora ya se nota una manifiesta tendencia al descenso (12), (11), precedida por un aumento inicial (5). (También nosotros observamos en 2 sujetos sometidos a tratamientos electroconvulsivos, a los quince minutos, un aumento del $54 \%$ sobre las cifras basales, para a las 2 horas notarse un descenso del $40 \%$ y a las cuatro horas del $69 \%$, como promedio).

En el histograma Ne 5 , hemos comparado las variaciones diurnas espontáneas obtenidas en el período de cuatro horas y las variaciones oblenidas en la altura; claramente se observa el mayor descenso en la altura. Pero debemos recordar que ambas determinaciones no fueron hechas en igual número de horas y a las mismas horas del día. Sin embargo, quizás nuestras cifras de la eosinopenia en la altura han podido ser aún mayores. La razón para ello está en que hemos obligado a nuestros sujetos a levantarse de 3 1/2 a 5 a.m., lo que de por sí constituye un stress. Transcribimos a Pincus y Hoagland (29). "El levantarse en la mañana hace tiempo que nos pareció un "stress" y hemos comunicado que la gente en general muestra una excreción aumentada de adrenocorticoides que ocompaña al inicio de lás actividades diarias. Por ejemplo, hay un aumento promedio del $50 \pm 14 \%$ en la excreción de los 17-Ketosteroides durante las dos primeras horas después do levantarse, sobre el nível del sueño; y esto llende a declinarse progresivamente durante el día".

Una observación sumamente interesante nos parece ser la que se refiere al descenso de eosinófilos en los sujetos que hicieron un cuadro de soroche agudo. Fueron ellos los que en el cuadro NQ 5, están signados con los números 7, 18, 20 y 21 . Tres de ellos hicieron un descenso de eosinófilos de más del $80 \%$ (vale decir el 


\section{Exingesposmat}
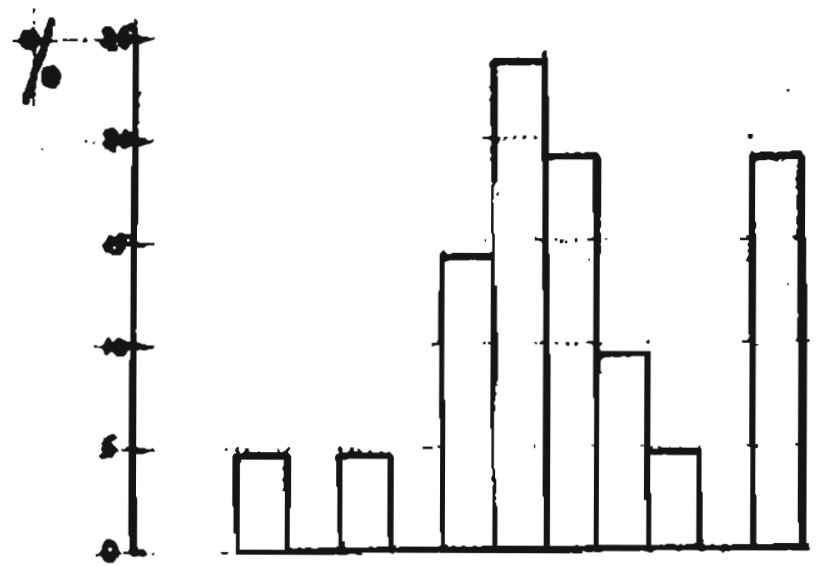

Ex Linn
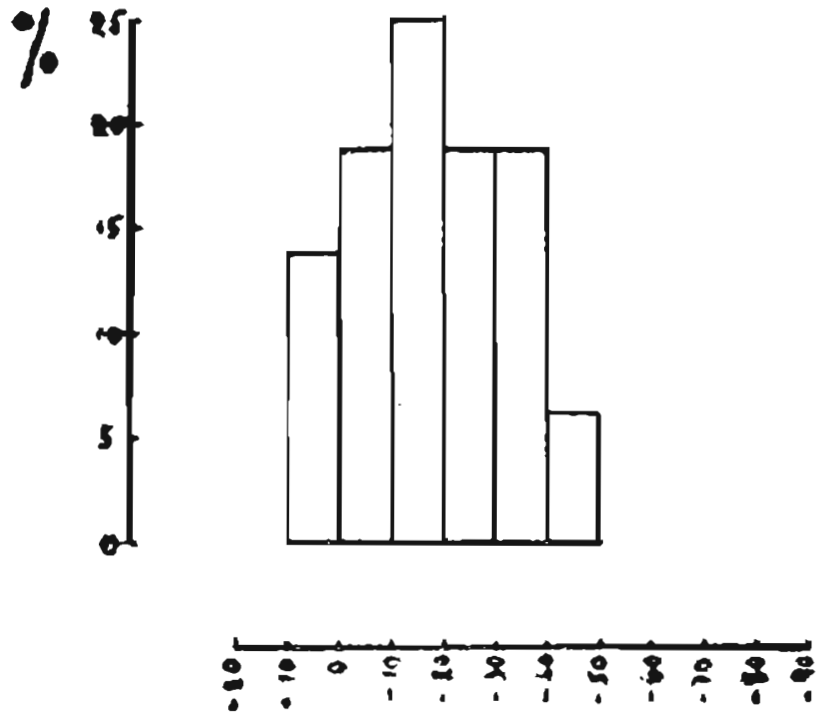

Eosinofilos \% 
$75 \%$ de los sujetos asorochados). Mientras que de las 17 personas réstarites sólo uno mostró una baja tän intensa. Si aceptämös "que la eosinopenia aquí es un fiel reflejo de la función estimulada de la suprarrenal, podríamos decir que en el sujeto asorochado ella está más excitada y que segrega mayor cantidad de $\mathrm{C}_{11}$ y $\mathrm{C}_{17}$ oxisteroides, causantes de esta baja de eosinótilos (39). Por lo que ya sea que esté el causal del soroche a nivel de los pulmones con la consigulente dificultad de adquisición del oxígeno atmostérico Monge (36), o más bien en los tejidos, donde haya un estorbo para su adquisición Hurtado (18), probablemente no sea una hiperfuhción insuficiente de la glándula suprarrenal (por lo menos en cuanto a la secreción de los 11-17 oxisteroides se refiere), la causante del cuadro ds soroche agudo.

\section{CONCLUSIONES}

Se ha investigado la respuesta suprarrenal por la prueba de los eosinófilos, en un grupo de sujetos, sometidos a la anoxia aguda, al llevarlos a la altitud (Morococha). Tamblén en forma com. plementariar, se han investigado los valores normales de los eosi: nófilos circulantes a nivel del mar (Lima), y su variación en 4 horas. Llegamos a las siguientes conclusiones:

$1^{\text {a }}$ - En la mayoría de sujetos sometidos a la anoxia aguda se produce un descenso en el número de eosinófilos circulantes, el que sería motivado por una excitación de la glándula suprarrenal en la anoxia aguda.

$2^{a}$-La mayoría de los sujetos que presentan soroche agudo, muestran una eosinopenia más intensa, en comparación con los no asorochados. Lo que indicaría una más intensa estimulación suprarrenal en los asorochados y una respuesta que parece estar de acuerdo con la intensidad del estímulo.

3a-El número de lẹ eosinófilos circulantes en 25 personas jó. venes, clínicämente normales, en ayunas, osciló entre 70 a 372 por milimetro cúbico, a nivel del mar.

$4^{a}$-Los eosinófilos varían espontáneamente en el lapso de 4 horas (de 8-9 a.m. a 12-1 p.m.), mostrando generalmente un descenso. 
I. Almeight r. and Reióenstein F. C.-The parathyroid glans and metabolic bone disease. Baltimore. Q. Williams and Wilkins, 1948.

2.-Altschule M. D. and Parkhurst B. H.-Effect of treatment on excretion of 17-Ketosteroids in patients with mental disease. Archives of Neurology and psychiatry. 64: $516,1950$.

3.-Artunkal S. and Kayahan S.-The effect of glucose on the circulating eosi.nophils. Journal of Clinical Endocrinology and Metabolism. 12: 1095, 1952.

4.-Best W. R. and SAMTER M.-Variation and error in eosinophil counts of blood and bone marrow. Blood 6: 61, 1951 .

5.-Bliss E. L., Rubin S. and Gilbert T.-The ellect of adrenalin on adrenal cortical function. Journal of Clinical Endocrinology. 11: 46, 1951.

6.-Cleghorn R. A. and Graham B. F.--Sstudies of adrenal cortical activity in psychoneurotic subjects. The American Journal of Psychiatry. 106: 668, 1950.

7.-Dougherty T. F. and White A.--lnfluence of hormones on lymphoid tissue structure and function. The role of the pituitary adrenotrophic hormone in the regulation of the lymphocyles and other celular elements of the blood. Endocrinology. 35: 1, 1944.

8.-Ellestad M. H. and Reed J.-Circulating Eosinophils in cardio-vasculat stress. Annals of Internal Medicine. 36: (Parl 2), 551.

9.-Fisher B. and Fisher E. R.-Observations on the eosincphil count in man. A proposed lest of adrenal cortical function. The American lournal of the Medical Sciences. 221: 121, 1951.

10. -Flink E. B. and Halberg F.-Clinical studies on eosinophil thythm. Journal of Clinical Endocrinology and Metabolism. 12: 922, 1952.

11.-Gabrilove J. L.-The Jevel of the circulating eosinophils following trauma. journal of Chinical Endocrinology. 10:637, 1950.

12.-Gramam B. F. and Cleghorn R. A.-Changes in the circulating leucocytes following electrically induced convulsions in man. The Journal of Clinical Endocrinology. 11: 1469, 1951.

13.- Hernández Morales F., Casas C. B. and García Sanz M.--Thorn test in patients with eosinophilia related to parasitic infection. J.A.M.R. 144: 379, 1950.

14.- Hartman F. A. and Brownell K. A.-The adrenal gland. Philadelphia: Lea and Febiger, 1949.

15. - Houssay R. H.-Las suprarrenales en la hipoxia y la intoxicación por hista. mina. Buenos Aires: El Ateneo, 1951.

16. - hiurtado A., Merino C. y Delgado Fesres E.-la influencia de la anoxamia sobre la actividad hemaiopoyética. Analos de la Facultad de Madicina. Tomo XXIX, $\mathrm{N}^{\circ} 2,1946$.

17. - Hurtado A.-Métodos Esladislicos. Analss de la Facultad de Mediana. Tomo XXVIII, 1945.

18. - Hurtado A.-Aspectos fisiolúgicos y potolúgíon de la vida en la altura. Lima: Euprese Editora "Rimac" S. A., 1937. 
19. - Ingle D. J.-The tunctional interrelationship of the anterior pituitary and the adrenal cortex. Annals of Internal Medicine. 35: 652, 1951.

20.-Kinsell L. W.-The clirical application of pituilary adrenocoticotropic and adrenal steroid hormonos. Annals of Internal Medicine. 35: 615, 1951.

21.-Kuhl W. L., Wilson H. and Rarli E. P.-Measurments of adrenal cotical activity in young men subjected to acute stress. The Journal of Clinical Endocrinology and Metabolism. 12: 393, 1952.

22. -Long C. N. H.-The concitions associated with the secretion of the adrenai corlex. Federation Proceedings. 6: 461, 1947.

23. -Mason II. L. and Engstrom W. W.-The 17-Kelosteroides: their origin determination and significance. Physiological Reviews. 30: 321, 1950.

24. - Monge C.-La entermedad de los Andes. Anales de la Faculiad de Medicina. Nos. 1 y 2, Abril a Julio de 1928.

25. -Monge C.-High altitude disease. Reprinted from the Archives of Interal Medicine. 59: 32, 1937.

26.-Penney R., Bedell Thomas C. and Lewis R. A.-Reduction in the number of circulating eosinophils following induced anoxemia. Bull. Johns Hopkins Hops. 86: 102, 1950.

27.-Penzmutter M., MUfson M,-The hupoglicemic and eosinodenic response to insulin. A test for Dituitary-adrenal insufficiency. The Journal of Clinical Endocrínology. 11: 277, 1951.

28.-Perloff W. H., LeVY L. M. and Despopoulos A.-The eosinophil responde to stress of patients with surgiral produced thalamic and hypothalamic lesions. The Journal of Clinical Endoctinology and Metabolism. 12: 36, 1952.

29.-Pincus G. and Hoagland H.-Adrenal cortical responses 10 stress in normal men and in those with personality disorders. Part 1. Some stress responses in normal and psychotic subjetds. The American Journal of Psychiatry. 106: $641,1950$.

30.-Pincus G. and Hoagland H.-Part II. Analysis of the pituitary-adrena! mechanism in man the American lournal of Psychiatry. 106: 651, 1950.

31.- Randolph T. G.-Blood studies in allergy: I The direct counting chamber delermination of eosinophils by propylene glycol aquos stains. The Journal of Allergy. 15: 89, 1944.

32.-Roche M., Thorn G. W. and Hilzs A. G.-The levels of circulaling eosino. phils and their response to ACHT in Survery. The New England Journal of Medicine. 242: 307, 1950.

33. Sayers G.-The adrenal cortex and homeostasis. Physiological Reviews. 30: $241,1950$.

34.-Selye H.-Textbook of Endocrinology. Montreal: Acta Endocrinológica Ine., 1949.

35. - Selye H.-The physiology and pathology of expousure to stress. Montreal: Acta Inc. Medical Publishers, 1950.

36.- Shands H. C. and Bartter F. C.-A statistical analysis of the "ACTH Test" changes in the eosinophil count in normal and in psychoneurotic subjects. The Journal of Clinical Endocrinology and Metabolism. 12: 178, 1952. 
37. Speirs R. S. and Meyer R. K.-The elfects os stress, adrenal and adrenocorflcolrophic hormones on the circulating eosinophils of mico. Endocrinology 45: 403, 1949.

38. -Tatror R. H., Gross M. and Rugy I. J.-Non convulsive electrostimulation and the pituitary-adrenocortical system. The Journal of Nervous and Mental Diseases. 114: 1951.

39.-Thorn G. W., Forsham P. H., Garnet Prunty F. T. and Hills A. G.-A test for adrenocortical insufficiency. The response to pituitary adrenocorticotropic hormone. J.A.M.A. 137: 1005, 1948.

40.-WEINER H. A. and Morkovin D.-Circulating blood oosinophils in acute infectious disease and the eosinopenic response. The American Journal of Medicina. 13: 58, 1952. 